\title{
Research on the Intelligentized Spreading of Short Videos
}

\author{
LI Zhongmin ${ }^{1}$ \\ ${ }^{1}$ College of Communication, Xijing University, Xian, China, 710123 \\ Corresponding author.Email: zhongminyn@163.com

\begin{abstract}
With the development and popularization of smartphones, short videos are seeing an explosive growth nearly everywhere. As a new form of mass media, which is easy to produce and fast to spread, short video has been enjoying a very special and important status in information communication and content and entertainment industries. With the advent of $5 \mathrm{G}$ technology, how will short video develop? To address this issue, this paper looks into the characteristics and current situation of short video, the features and forms of its intelligentized spreading as well as the problems and trends of its future development.
\end{abstract}

Key words: Short video, Intelligentized spreading, $5 G$

\section{短视频智能化传播探究}

\begin{abstract}
李忠民
${ }^{1}$ 西京学院传媒学院 中国 西安 710123

Corresponding author. Email: zhongminyn@163.com

摘要: 随着智能手机的发展和普及, 短视频作为一种新的大众化的传播形式随处可见, 易制作、快传播的特性, 决定了它在社会信息传播和内容产业中的特殊地位。由于其独特的传播优势, 已经成为人们生活娱乐的重要组 成部分。随着 $5 \mathrm{G}$ 时代的到来，短视频有什么样的发展？本论文对短视频的特点和现状、智能传播的特征、表 现形式、和它在发展中存在的问题以及未来的发展趋势进行了分析和探讨。
\end{abstract}

关键词: 短视频; 智能传播; $5 G$

\section{1. 引言}

智能手机的不断普及和AI技术的广泛应用, 新的 媒体平台正在走向社交、视频化、智能化。到了 $5 \mathrm{G}$ 时 代，这些表现也越来越明显。

近年来, 无论是实践方面还是理论方面, 短视频 不仅仅是一个热词, 就传播形式而言, 短视频在互联 网上非常火爆, 几乎是所有媒体的最好诠释者。它可 以包含在所有媒体和传播渠道中, 用生动、方便、直 观的方法, 全面展现社会生活的各个方面和细节, 成 为人与社会以及人与人之间的一种新连接方式。就产 业发展而言, 短视频正以强势夺取用户注意而占据市 场份额, 持续受到资本支持, 其制作成本小、正从草 根化向专业化、品牌化方向转变, 带动了网红经济的
兴起，催生了多元化的增值服务模式，建立了 “短视 频+”的产业链崭新格局, 也成为新媒体推进融合的 必不可少的渠道和方法。

\section{2. 短视频传播特点及发展现状}

\section{1. 短视频的特点}

短视频的短是指视频的内容短, 也可以称为短片 视频, 是近年来急速发展的因特网的新业态, 也是互 联网传播方式中内容的一种表现形式。其长度大多是 在数秒, 有的最长不超过 5 分钟。短视频利用视频信 息相较文字图片媒体独有的优势，以及 “短平快” 的 特性, 一方面使得受众有了更好接收信息的体验, 另 一方面, 给视频平台引来大量流量, 深受各大平台、 
粉丝和资本的青崃, 得到了喷射式的发展。它的主要 特点有：

\section{1. 1. 内容丰富, 多元化}

短视频作为媒体信息的承载方式，其内容丰富多 彩，涵盖了音乐、舞蹈、美食、运动、科技、时尚、 汽车、旅行、亲子、影像、政务、剧情、动物、创意、 传统、动画、生活等人们日常生活的各个领域。特别 是自黑搞笑类、一见钟情类、温暖治愈类、酷炫技术 类、情感共鸣类等短视频内容, 独占鳌头, 更能获得 用户青睐，更容易成为爆款。

\subsection{2. 碎片化、高传播、低门槛}

这里的碎片化是指短视频的种类非常多, 内容本 身的碎片化, 传播短视频的媒体介质的碎片化, 受众 人群的碎片化, 用户利用碎片化的时间观看短视频。 高传播是指短视频在 $5 \mathrm{G}$ 时代传播速度非常之快。低门 槛是指自媒体时代短视频的制作成本低，技术要求低， 非专业大众就可以制作发布传播短视频。

由于短片视频产品、高传播、低门槛特性, 目前 短片视频整体移动互联网用户渗透率较低, 用户奖金 依然存在, 用户发展空间较大。中国的短视频产业链 参与主体不断丰富, 产业生态逐渐强健。

\section{1. 3. 有吸引力、高粘性}

由于用户对于内容的要求和品位也越来越高, 因 此垂直领域的短视频将受到热捧, 基于大数据统计和 深度学习, 收集用户的收看习惯数据, 推测用户喜好, 为用户推荐短视频内容, 做到真正意义的千人千面和 个性化精准推荐, 使短视频具有强吸引力和高粘性的 特点。

短片视频的吸人、吸时、吸金、吸睛等特征, 使 受众的粘性增强, 是广大用户日均使用时长最长的视 听产品, 正在渗透到电商、教育、直播等各个领域。

\section{2. 短视频发展现状}

日前, 短视频迅猛发展, 积极与各行各业相结合, 拓宽短视频生命力, 成为继移动通信之后的第二网络 应用, 在使用户下沉的同时, 成为互联网的底层应用。

据中国网络视听节目服务协会发布的《2020中国 网络视听发展研究报告》显示, 截至2020年6月, 中国 短视频用户达到 8.18 亿, 网络直播用户达 5.62 亿, 有 近九成网民使用短视频。

从发展格局来看: 第一梯队的抖音短视频和快手 两强格局持续强化, 第二第三梯队如雨后春笋般生长 起来的各类短视频平台, 如西瓜视频、微视、土豆视 频等竞争激烈。
从用户角度看: 短视频用户以 80 后、中低学历居 多, 因此短视频在用户下沉中具有相当影响力。

从短视频使用情况看: 人均单日使用时间长达 110 分钟，成为个人使用时间最长的媒介形式。

从短视频内容分析来看: 短视频深入生活, 承担 多元角色，美食、影视、音乐、教育、科技等等都成 为短视频内容的新标签, 垂直领域依旧不断地精细化 发展。【1】

\section{3. 短视频的智能化传播特征}

\section{1. 全民主动性、参与性增强}

首先，短视频的用户既是观众也是主角。他可以 刷看别人的视频, 并收藏和下载, 也可以发布自己的 录像短视频。分享自己有趣的生活片段和独特的技能, 甚至当时发生的新闻、独特的见闻。短视频应用为用 户建立了双向交流平台。在这里发布者可以得到阅览 量、赞词等反馈。观众点赞、分享、收藏、转发自己 喜欢的视频，促进短视频的制作，产生大量的流量数 据。这种方便的自制性和低成本制作门槛使用户从被 动接受过渡到主动创新, 喜欢从生活中汲取灵感创作、 传播, 提高了参与的积极性和主动性。

第二, 短视频应用不断在推出新的玩法, 例如: 2021年春节期间，抖音平台利用 “看视频”、“拍新 春视频” 增加点亮 “团圆家乡年” 灯笼次数, 来集齐 “祈福灯笼”, 得现金红包。另外, 也有一些短视频 APP与直播平台相结合, 进行产品合作, 直播网红利 用短视频平台的社交功能与粉丝联系。短视频APP直 播平台, 还利用奖金红包、打赏、广告等方式实现成 熟的变现能力。

\section{2. 碎片化时间，不受时间空间限制，互动性 增强} 过程中。

这一特征主要体现在短视频的内容生产和传播

首先, 就新闻短片而言, 它的应用的兴起不仅仅 是媒体的生产, 而是把新闻生产的能力委托给参加新 闻短片应用的用户。任何短视频用户都可以作为公民 记者登场，打破了以往传播媒体必须第一现场出现的 空间和时间限制。同时, 高速网络的新闻传播, 使新 闻的时效性变得更强, 每个用户都可以在第一现场传 达最新的报道，使事发现场第一时间得以呈现。

其次, 视频有着比文字图片更先天的优势。短视 频利用视觉和听觉的冲击和形象感染力, 使观众短时 间内受到强烈刺激。与其他传统的文字相比, 用户在 汶览信息时, 可以不从一开始读到最后, 花了很长时 间读完文章。和传统的长视频媒体相比, 不需要看整 段的视频才能明白。由于时间限制, 这促使UGC ( User-generated Contest, 用户生产内容)，在制作 
时去取其精华。不花太多时间，适合移动互联网用 户的零碎使用习惯, 实现符合现代人快节奏的生活 方式。

再次, 和其他视频产品相比, 比如: 纪录片、电 影、综艺节目、甚至电视剧等。短片视频不需要太长 的时间来积累画面感情, 而是直接呈现最有趣, 最高 潮的部分。为了与用户共享, 短视频应用迅速夺取了 人们的碎片化注意力。

另外, 随着互联网技术的发展, 短视频等流媒体 的播放速度不断提高, 流量费用降低, 也不受时间、 空间的限制, 随时都可以播放。这也促进了短视频和 日常生活的深度的结合。在碎片化的时间里, 用户想 利用短暂的时间, 了解生动的立体信息, 还可以随时 与发布者或其他受众交流互动，陈述自己的观点见解。 短视频的这一特点更好地满足受众碎片化时间需求, 更容易碎片化的消费和社会化的传播。

\section{3. 受众群体呈现年轻化}

短视频平台的接受者呈现出年轻化的特征。根据 CBNData提供的数据, 30 岁以下的青年用户是短片接 受者的主力, 用户使用的粘性也更高。在土豆平台上 30 岁以下的人超过 $60 \%$, 其中 $19 \sim 30$ 岁的人特别突出。 他们对音乐、舞蹈、视频剪辑有着极高的热情和创造 力, 他们追求自由的生活态度, 有个性、喜欢新潮。 用户还可以通过音乐短视频依据自己的特色和个性 进行再次创作。对于很多观众来说, 有的通过 “看短 片视频” 可以取代实际的社会体验和实践, 可以认识 他人和社会, 享受第一视点的主观体验。由于社会发 展的不平衡, 有些实际体验不到的年轻人即使坐在家 里也能了解 “天下事”。也有一些年轻人想通过 15 秒 短视频, 怀揣网红梦想, 他们乐于拍摄短视频, 以展 示自己、表达自我。

\section{4. 智能推送与跨平台的传播}

短视频的传播, 主要通过社交共享生活等平台传 播。手机微信、微博、抖音、快手、小红书等平台是 短视频的重要载体。用户通过碎片化时间汶览, 平台 智能推送, 满足受众的各种需求。同时, 这些社交平 台在PC终端也有网站、应用app等, 短视频资源可以 同时在多个平台上发布并普及。用户只要拥有设备, 就可以随时播放。为了更好满足用户的需求, 短视频 的传播也具有跨平台、跨设备传输的特点。[2]

\section{4. 短视频智能化传播的表现形式}

智能传播是 “以新能源、人工智能、大数据、物 联网、机器人技术等技术发展和突破为基础, 将物联 网数据流替代人力、技术、传统生产要素, 以智能技 术决定内容的生产、传播、营销与一体化为决策方向 的新传播方式” [3]。也有学者说, 智能传播是以 人工智能技术来 “感知环境、获得知识并使用知识”
来实现信息生产活动。(孙江, 何静, 张梦可, 2020)

【4】也就是说, 智能传播是将人工智能技术应用于 信息的生产和普及的新的传播形式、体系和生态。它 与传统传播、互联网传播相比, 无论是在信息丰富程 度、传播模式, 还是新闻传播的透明度、及时性、交互 性等方面, 有着明显的优势: 在信息过载时代, 智能 传播实现了精准传播; 提高了传播效率; 可以对用户 实施多点对一点的信息传播; 高度的透明打破了信息 传播的不对称; 最大程度地实现了信息传播的交互性 和及时性。

那么, 作为新媒体的短视频是如何进行智能化传 播的?

目前, 短视频智能传播主要表现在短视频内容生 产的自动化和短视频产品推送的精准化, 二者颠覆了 视频生产与分发的传统模式, 解决了信息过载, 重建 了用户连接。然而, 自动化生产短视频内容和为用户 画像实现精准推送都离不开大数据算法的支撑。

\section{1. 短视频内容生产智能化}

自动化的短视频新闻消息的生产过程是 “自动算 法过程”, 该自动算法过程在无或有限的人类参与下， 通过预先设置的程序将数据转换成新闻消息。内容生 产者在掌握大量数据的基础上, 参照某些计算公式, 运用算法, 套用已有模板生成规范的新闻视频; 或者 将采集到的数据与某个记者的数据模型相结合, 通过 运算生成符合该记者风格的短视频新闻。

目前的网络短视频生产主要分两大类, 一种是 专业内容生产的 PGC (Professionally-generated Content）短视频, 另一种是用户内容自主生产的UGC 的短视频。伴随着市场竞争的越来越激烈, “ PGC+UGC” 的短片生产模式也形成了新的趋势。无论 是怎样的内容生产模式, 都在智能化的推进。

例如, MAGIC的短视频智能生产平台, 它能帮助用 户完成更高效短视频内容的创作。提供在线视频的智 能编辑、智能字幕、直播剪辑、云素材仓库、一键全 网发布、团队合作等功能, 大大提高了短视频的制作 效率。用它创作的短视频更加智能, 其拥有的智能数 据工坊、智能媒体资源平台、智能生产引擎和智能主 题, 使智能时代的 “原油”数据在AI催化下得以提纯, 把算法处理的素材 “零件” 在储存仓库中随时被调用 装配，这样新闻短视频生产就像 “乐高” 工业流水线 式高效生产，以灵活高效的智能化模版，全面释放生 产力。MAGIC可以在短时间内生产短视频, 最快只需 要6秒。MAGIC可以批量生产短视频。平均日产量能达 到10000条。MAGIC在2018年世界杯期间生产的视频总 播放量突破了一亿次。新华社使用MAGIC, 在2018年中 国国际进口博览会上生产 554 部视频, 最快的是 13 秒 完成。将近3000家媒体入驻当时的云平台, 通过MAGIC 高效的进行短视频生产【5】。 
又比如:「来画」视频创作者联盟。它是一款视 频创作营销平台。作为一家云端商业级短视频智能创 作平台，「来画」完全摒弃了MAYA、AE等专业动画制作 软件中晦涩难学的部分, 仅保留高频使用的创作功能, 可在短时间内生成一个有趣的动画短视频。丰富视频 模板素材免费用，对于企业用户，还有动画高端定制 服务。人性化的功能配以友好的交互设计, 再加上涵 盖了图片、字幕、音乐、视频等媒介的海量商用素材 库, 进一步降低短视频制作的门槛和成本, 使用户真 正实现了像做PPT一样制作短视频【6】。

智能技术的发展是UGC短视频制作的前提, UGC的 核心之一又是 “去专业化”。它在内容的生产和共享 的全过程中, 可以不要求有专业人员的参与, 整个视 频内容制作都是业余化的。

目前有多种大众的、适合零基础人很快掌握的短 视频编辑制作软件, 比如, 剪映, 快手、抖音等, 生 产者利用智能手机或其它摄影摄像器材拍摄, 将素材 导入编辑软件中, 利用嵌入的视频模板剪同款, 或者 自己进行剪切, 加特效、字幕、音乐等非常方便、快 捷、智能化创作出较高质量的个性化短视频。

由此可见, 无论采取何种方式, 算法几乎介入了 内容生产的各个环节, 通过大数据的云计算与分析, 自动生成了新闻短片, 大大提高新闻生产的速度和产 品的数量, 并有效消除人为的技术性差错。

\section{2. 短视频产品推送精准化智能化传播}

\section{2. 1. 什么是大数据精准推送?}

精准推送多是指新媒体根据对大数据的信息化 处理从而将人们想看的新闻、视频、段子、商品等置 于优先位置。简单来说, 就是根据不同用户使用、观 看、汶览、购买等一些操作进行大数据分析, 之后精 准推送给想要使用、观看、浏览、购买等操作的用户, 该推送经大数据分析, 很大程度上会是用户想要的内 容。推送是通过推送算法实现的, 目前, 推荐算法已 经成为国内外大多数新闻APP和社交媒体平台使用的 主流, 通过机器学习持续关注用户的阅读数据, 再辅 助以场景分析, 来提升视频新闻推送的准确率。推荐 算法的实质就是大数据分析, 通过抓取和分析用户的 人文数据和行为数据, 推测出用户的个人特征、阅读 偏好等。算法不仅提高了内容生产与信息推送的效率, 而且实现短视频与用户的精准匹配。

\section{2. 2. 短视频时代大数据精准推送的应用}

大数据应用的兴起, 快速发展的智能技术, 以及 算法推荐使得短视频等信息定制化、资讯分众化得到 更加广泛的应用。在智能手机等新媒体的普及后, 短 视频精准推送变得数据更准确, 更加全面, 范围更广, 受众更多。近年来, 新媒体时代的兴起, 各短视频平 台都吸引了大量的用户, 短视频行业的用户量的持续
增长, 其中用户个性化精准推送起到了关键性的作用。 在大数据、算法的作用下, 短视频平台根据用户的个 人喜好, 为每个用户都制定个性化推送服务, 让用户 持续看到自己想要的内容。接下来, 以抖音的平台为 例, 让我们来看看平台如何精准推送短视频吧。

抖音的AI算法是根据用户的喜好与需求而推荐 相应的内容的, 通俗地讲, 也就是推荐用户喜欢的东 西给用户看, 这看似简单, 其中蕴含的大数据与智能 算法推送却是极其深奥的。抖音算法的推荐标准是遵 循五个数据进行考核推荐的, 这五个推荐的数据量分 别是: 视频粉丝增涨量、视频点赞增涨量、视频评论 增涨量、视频播放增涨量、视频转发增涨量。只要达 到了这五个数据的基本数量, 也就是基本线, 那么抖 音的AI算法就会给与这个短视频基本的流量推荐, 然 后再根据这个给与的流量推荐所得出的数据再进行 综合判断这个短视频是否值得下一轮的推荐, 如果值 得, 算法就会继续给你流量分发与推荐, 进入高一级 的流量池, 再根据那五个数据量高一级的标准再运算 再衡量, 再分发与推荐, 短视频被推荐的越多, 传播 的就越广。如果不值得, 就会停止推荐, 那么这个短 视频就相当于被雪藏了。

通过算法精准推送满足了人们多元化、个性化的 短视频需求。通过智能化、定制化的信息传播机制, 实现了用户与短视频准确快速的匹配, 大大降低了信 息传递和获取的成本, 为人们生活娱乐带来了便利。

算法的推荐在高效便利的同时, 也出现了一些低 俗、劣质的视频信息推送, 引发诸多乱象。

\section{5. 短视频智能化传播应用中存在问题及未来 发展趋势}

\section{1. 短视频应用存在的问题}

\subsection{1. 过分重视视听效果, 无视其内涵建设, 泛娱乐化问题突出}

现阶段在短视频APP中充满了各种模仿内容, 他 们刻意追求视听效果, 重流量而轻质量。也就是说, 盲目迎合追求受众感官刺激的心理，仅重视频特效技 术的开发和漂亮的画面的制作, 忽视视频的内容本身, 虽然不断出现爆款, 但缺乏内容创造性。例如, 某些 平台网红 “小姐姐” 的舞蹈, 受众得到了浅薄的感官 刺激, 在短时间内虽然会带来大量流量, 但是同质化 无内涵的表演不会带来真正的影响力。

\section{1. 2. 非专业内容生产者占主导}

智能手机和数码相机等智能化摄录工具, 使高智 能、低门槛、低成本短视频制作趋向于非专业化的水 平和质量。我们经常接触的短片内容尽管多种多样, 但是，大家多展现的是日常生活琐事和娱乐搞笑类， 并且由于使用门槛低, 拍摄又有时常限制, 所以视频 
内容拍摄大多不具备专业性和技术性。视频的质量也 是鱼龙混杂。一些短视频创作平台在商业利益驱动下, 盲目迎合大众心理, 这种娱乐化倾向越来越明显, 甚 至出现低俗混乱的倾向。

\section{1. 3. 监管难度大，造成不可低估的负面效应}

一方面, 由于短视频的生产快，传播迅速，平台 本身监督管理容易顾此失彼, 容易滋生部分消极的影 响青少年身心健康的信息, 影响社会稳定。另一方面, 碎片化视频传播, 容易造成诸多抄袭和版权问题，传 播过程中的侵权问题也很难去界定，监督管理的困难 很大。隐私受到侵犯, 正常生活受到网络暴力的影响, 网络空间充斥了负面能量, 对许多网民和社会造成不 可低估的负面影响。

\section{1. 4. 影响青少年的价值观}

短片视频传播热, 一方面为网红提供了平台和机 会。同时, 网络红人的大量出现也表现出短视频行业 的过度娱乐化倾向。因此, 低质量的网红易出现 “闪 崩” 现象, 这在短视频领域常常发生。有些青少年为 了当网红受到追捧, 不惜倾其财力, 甚至走不正当渠 道, 从而影响正确的价值观。另一方面, 大量娱乐化 的内容中, 有不适合青少年身心健康的, 特别是在培 养青少年价值观的关键时期, 如果看不适当的内容, 对青少年有害无益。此外, 短视频应用程序的设置会 产生用户行为的惯性, 青少年沉溺其中会浪费很多宝 贵时间，影响青少年学业的发展。【7】

针对目前短视频应用存在的问题, 以及 $5 \mathrm{G}$ 时代带 来的红利, 短视频的生态发展未来前景又将如何?

\section{2. 短视频末来发展趋势}

据官方发布，短视频成为继即时通信之后的第二 网络应用, 有 $60.4 \%$ 的受众每天都看短视频。与综合 类视频 (网络剧、电影、综艺节目)、网络直播、网 络音频等相比, 短视频是占比最多和增速最快的视听 领域。短视频平台迅速实现了市场规模的扩大, 以抖 音、快手等为代表的短视频APP吸取了大量的用户。 抖音的日活跃用户在 2020 年 8 月突破 6 亿人, 到 2020 年 12 月为止平均每天的视频搜索量突破了 4 亿, 就连快 手的日活跃用户也有 3 亿人次。

结合自己的实践以及资深从业者对音视频行业 发展认知，未来的短视频有以下 6 个发展趋势。

\subsection{1. 短视频将推动市场格局变化}

根据《2020中国网络视听发展研究报告》，截至 2020年6月, 短视频用户的使用率最高达到 $87.0 \%$, 用 户规模是 8.18 亿。综合视频用户使用率 $77.1 \%$, 用户规 模是 7.24 亿。网络直播使用率是 $59.8 \%$, 用户规模是 5.62 亿。网络音频用户使用率稍低, 占 $29.3 \%$, 用户规
模是 2.75 亿。在今后 1 至 2 年内, 短视频的渗透和深化 将持续。通过扩大布局直播, 对微剧普及推广, 加入 综合视频内容以及入口拓宽版图。CCTV新媒体占据塔 尖, 抖音快手和㗪哩㗪哩占第二层, 腾讯和爱奇艺等 深耕发展, 它们共同成为网络视听行业的大佬, 推动 了短视频市场格局。

\subsection{2. 短视频从泛娱乐化向垂直化和资讯性 发展, 内容精品化趋势越发明显}

未来仍然是以 “内容为王”。如果想让短视频继 续大热火爆, 在确保制作品质量的基础上, 必须不断 进行内容创新。优质创新的内容才是短视频应用提高 用户粘性的关键。5G时代的网络速度为传播更清晰的 短视频创造了条件, 因此, 精品内容的短视频将成为 未来短视频传播主要方式。垂直领域的专业内容的制 作者将越来越多, 高质量, 有特点, 有创新的专业短 视频将会蓬勃发展。

从内容不断细分到如今热门短网剧、短视频因其 强共情、精制作频上热搜，可以看出，短视频的内容不 再仅以猎奇、娱乐抓住人们的视野, 内容的精品化发 展成为短视频内容开发者关注的重点。突破单一娱乐 风格, 推进精品制作, 体现人文关怀, 才能突出优质 内容的价值，打造短视频的核心竞争力。【8】

\section{2. 3. 技术赋能创新，融合各平台优势}

“视听+”将成为行业发展的新方向，同时5G技术 催生短视频等视听新形势的发展。在 $5 \mathrm{G}$ 时代, 相信先 进的拍摄技术、强调功能的剪辑软件等也将逐渐匹配 升级，目前短视频拍摄技术正向虚拟和全景式演进。 比如，提供独特视角的无人机技术; 加强用户沉浸的 虚拟现实 VR; 信息量宏大的全景技术。又如，腾讯生 产的微视周边成套产品、微视专用摄像等装置, 都为 短视频的智能传播提供技术支持和技术创新。同时， 技术的赋能创新, 也为短视频跨平台融合发展搭建了 桥梁。【9】

\section{2. 4. 监管机制更加完善，推进规范化运作}

在未来短视频的发展中, 出台相应完善的政策规 范, 优化发布内容审查机制, 是加强管理和把关的基 本保障。一方面, 短视频app后台监督管理机制会越 来越规范，用户举报机制和内容自我审查机制也更加 准确有力。另一方面，政府会出台相应更完善的法律 法规政策，规范保护短视频的知识产权，推进短视频 app平台的规范化运营，保障短视频向正规健康方向 发展。 


\subsection{5. 短视频与网络直播将成为网业主要增 量}

根据《2020中国网络视听发展研究报告》，短视 频市场增加达到 $85.5 \%$ ，网络直播市场增加为 $67.1 \%$ 。 相比于影视剧、纪录片和网络音频, 未来依旧会有更 大部分的用户导向短视频和网络直播。

\subsection{6. 短视频的商业模式增强}

短视频作为近几年来内容创业领域最大的风口, 其信息量大、场景丰富、传播广等特点备受广大用户 的推崇, 巨大的传播价值让短视频迅速晋级成为又一 大商业宣发渠道。

短视频的流量可以利用电商和广告等形式进行 变现, 探索出新的变现方法。现阶段新的商业变现方 式还只是简单直接的形式, 通过单纯地植入广告来实 现, 但用户的活跃度是维持平台生命力的根本原因, 用户的活跃度带来商业的变现, 不断流入平台的资金 链又使平台有新的发展。平台设计者需要把控的是, 巧妙地保持用户与商业变现之间的动态平衡, 目前这 种简单的变现方式不能满足平台和用户的需求, 随着 短视频的未来发展, 商业的变现方式也随之变化。现 阶段有许多短视频平台的短视频, 不再是以往的艺术 观赏、娱乐、教育、社会价值，而是体现其商业价值。 短视频产品通过视频推荐到达用户，用户汶览观赏之 后, 关注、点赞、评论、分享、转发, 再通过系统平 台推送到下一级流量池, 关注的粉丝越来越多, 甚至 递增。短视频产品有的通过粉丝打赏变现, 也有的通 过开商品堐窗、直播带货等方式, 形成一条短视频产 品商业链。

短视频作为一种新的媒体媒介, 可以给很多行业 注入新的活力。现在的行业还处于摸索商业化道路的 初期, 行业价值必须进一步挖掘。随着短视频平台更 加规范化发展, 短视频产品内容质量逐渐提高, 短视 频与各行各业的融合度愈来愈深, 市场规模也越来越 大，保持高速增长的良好态势。

\section{6. 结论}

综上所述, 随着 $5 \mathrm{G}$ 、云计算、移动互联网等新通 信技术的快速发展, 以及新一代智能终端产品的应用, 人类的信息传递系统已经进入了移动化、数字化、交 互化和智能化的新纪元, 这也顺应了时代的浪潮。无 论是各类主媒体平台, 还是各种娱乐社交平台, 正在 合力开启一个短视频的智能化传播的新时代。同时, 短视频发展还面临内容同质化、泛娱乐化、非专业内 容生产者主导、监管困难大、部分短视频影响青少年 价值观等问题也在发展中不断得以解决。回归生活化、 IP化和部分专业化生产, 也是对短视频行业长期健康 发展有益的尝试。相信短视频在各种平台共享和传播, 将重新走向发展的快车道, 它的智能化传播在未来生
活的诸多方面将产生深远影响, 形成新的 “短视频生 态”。

总之, 短视频虽短, 但是短视频智能化传播的生 命力很强, 其未来发展道路很长。

\section{REFERENCES}

[1] China Internet Audio-visual Development Research Report 2020: Insight into Industry Development and Future Trends. Retrieved from https://www.thepaper.cn/newsDetail_forward_955 3462.

[2] Xia YongFang. Research on the Current Situation and Development Trend of Short Video in the Mobile Internet Era [J]. New Media Research, 2017(12),p110-111.

[3] Meng Wei. Ethical Questions in the New Communication Scene [J]. New Media, 2018,(4),p46-47.

[4] Xu Xiangdong,Wang Yixi. Causes, Effects and Countermeasures of Algorithmic Bias in Intelligent Communication .J. Chinese Journal of Journalism \& Communication, Vol. 42 (10),2020,p 69-85.

[5] How Long Does It Take to Produce a Short Video? As Fast As 6 Seconds! Retrieved from https://www.sohu.com/a/285127285_1818842.

[6] Aiming at Content Dissemination in the 5G Era, "Laihua" Builds a Commercial-grade Short Video Intelligent Creation Platform. Retrieved from https://36kr.com/p/1724347318273.

[7] Huang Chuxin., Ren Fangyan. The Current Situation, Problems and Trends of Short Video Development in China[J]. News Tribune, 2017(5),p 26-31.

[8] Fan Lin. The Characteristics and Application of Short Video Communication in the New Media Era [J]. New Media Research, 2018(12),p113-114.

[9] Zhang Haojing. Analysis of the Current Situation and Future Development Trend of Mobile Short Video[J].Shenzhou， 2019(1),p 238-239. 